\title{
Giant Quantum Hall Plateau in Graphene Coupled to an InSe van der Waals Crystal
}

\author{
Z. R. Kudrynskyi, ${ }^{1}$ M. A. Bhuiyan, ${ }^{1}$ O. Makarovsky, ${ }^{1,}{ }^{*}$ J. D. G. Greener, ${ }^{1}$ E. E. Vdovin, ${ }^{1,2}$ Z. D. Kovalyuk, ${ }^{3}$ \\ Y. Cao, ${ }^{4,5}$ A. Mishchenko, ${ }^{4}$ K. S. Novoselov, ${ }^{4}$ P. H. Beton, ${ }^{1}$ L. Eaves, ${ }^{1}$ and A. Patanè ${ }^{1, \dagger}$ \\ ${ }^{1}$ School of Physics and Astronomy, The University of Nottingham, Nottingham NG7 2RD, United Kingdom \\ ${ }^{2}$ Institute of Microelectronics Technology and High Purity Materials, RAS, Chernogolovka 142432, Russia \\ ${ }^{3}$ Institute for Problems of Materials Science, The National Academy of Sciences of Ukraine, \\ Chernivtsi Branch, Chernivtsi 58001, Ukraine \\ ${ }^{4}$ School of Physics and Astronomy, University of Manchester, Oxford Road, Manchester M13 9PL, United Kingdom \\ ${ }^{5}$ National Graphene Institute, University of Manchester, Manchester M13 9PL, United Kingdom
}

(Received 6 June 2017; published 10 October 2017)

\begin{abstract}
We report on a "giant" quantum Hall effect plateau in a graphene-based field-effect transistor where graphene is capped by a layer of the van der Waals crystal InSe. The giant quantum Hall effect plateau arises from the close alignment of the conduction band edge of InSe with the Dirac point of graphene. This feature enables the magnetic-field- and electric-field-effect-induced transfer of charge carriers between InSe and the degenerate Landau level states of the adjacent graphene layer, which is coupled by a van der Waals heterointerface to the InSe.
\end{abstract}

DOI: 10.1103/PhysRevLett.119.157701

Indium selenide (InSe) is now a well-established member of the family of van der Waals ( $\mathrm{vdW}$ ) crystals with physical properties that differ significantly from those of graphene, hexagonal boron nitride ( $\mathrm{hBN})$, and transition metal dichalcogenides [1-4]. Recent work has demonstrated the potential of InSe for electronics and optoelectronics due its high electron mobility and photoresponsivity [5-8]. For example, field-effect transistors (FETs) that incorporate few-atomic-layer InSe encapsulated in hBN can achieve areal carrier densities approaching $10^{13} \mathrm{~cm}^{-2}$ with electron mobilities $\mu$ exceeding $10^{3}$ and $10^{4} \mathrm{~cm}^{2} \mathrm{~V}^{-1} \mathrm{~s}^{-1}$ at room and liquid helium temperatures, respectively, thus enabling the observation of Shubnikov-de Haas oscillations and the quantum Hall $(\mathrm{QH})$ effect [8].

Here we demonstrate that InSe can be employed in graphene-based FETs as an electrically active capping layer to "modulation-dope" graphene, thus leading to a "giant" $\mathrm{QH}$ effect plateau. Low-temperature measurements of the magnetoresistance of this InSe-capped graphene FET reveal the presence of a well-defined $\mathrm{QH}$ plateau at a filling factor $\nu=$ 2 in the magnetic-field-dependent Hall resistance $R_{x y}$. The plateau extends over a wide range of magnetic field $B$ from $6 \mathrm{~T}$ to a maximum available field of $14 \mathrm{~T}$. A giant $\mathrm{QH}$ plateau has been reported in epitaxially grown graphene on $\mathrm{SiC}$, a two-dimensional electron gas system that is likely to replace III-V compound heterostructures in the next-generation QH resistance standards [9-14]. Our measurements

Published by the American Physical Society under the terms of the Creative Commons Attribution 4.0 International license. Further distribution of this work must maintain attribution to the author(s) and the published article's title, journal citation, and DOI. demonstrate that this quantum magnetoresistance phenomenon is not unique to graphene on $\mathrm{SiC}$. We show that the close alignment of the conduction band (CB) edge of InSe with the Dirac point of graphene enables the magnetic-field- and electric-field-effect-induced transfer of charge carriers between InSe and the degenerate Landau level states of the adjacent graphene layer, which is coupled by a vdW heterointerface to the InSe.

For these studies, we prepared devices in which a freshly exfoliated flake of $\gamma$-polytype InSe is placed upon a lithographically formed section of graphene, configured as a multiterminal Hall bar with four pairs of Hall voltage probes; see Fig. 1(a). Single-layer graphene was grown by chemical vapor deposition (CVD) on a copper foil substrate and was transferred to a $\mathrm{SiO}_{2} / n$-Si substrate. The graphene was shaped into a Hall bar geometry using conventional electron-beam lithography and oxygen plasma etching. The $\mathrm{Au} / \mathrm{Ti}$ electrodes $(100 / 10 \mathrm{~nm})$ were formed by means of electron-beam lithography, metal evaporation, and lift-off. The InSe flakes were exfoliated onto a polydimethylsiloxane membrane from a bulk Bridgman-grown crystal. A selected flake was dry transferred to the graphene Hall bar, capping the graphene channel but not contacting the $\mathrm{Au} / \mathrm{Ti}$ electrodes. The layer of InSe forms a cap over one-half of the device to produce an InSe/graphene heterostructure in the form of a Hall bar. The voltage probes on the uncapped half of the graphene layer provide us with a "control sample." The two halves of the device are in a "series" resistance configuration with the same electrical current flowing through both of them. This allows us to study how the InSe capping layer changes the electrical properties of the underlying graphene. We have examined a series of devices with InSe layers of thickness between 10 and $50 \mathrm{~nm}$, all showing a common behavior. 


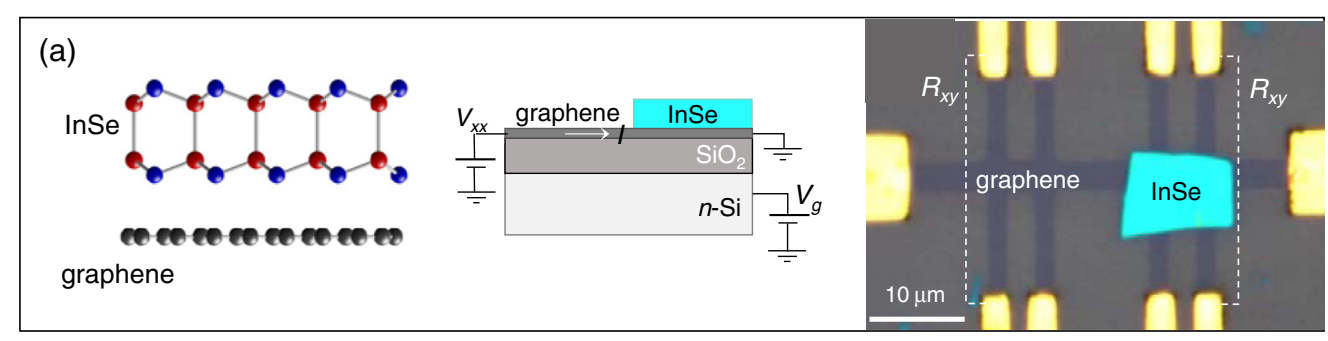

(b)

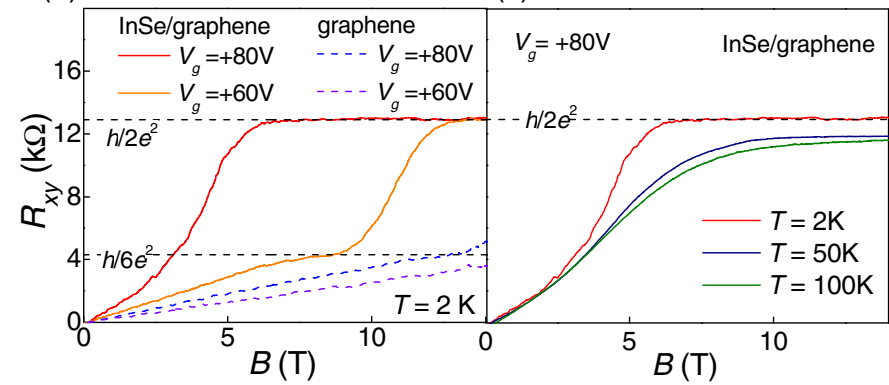

(d)

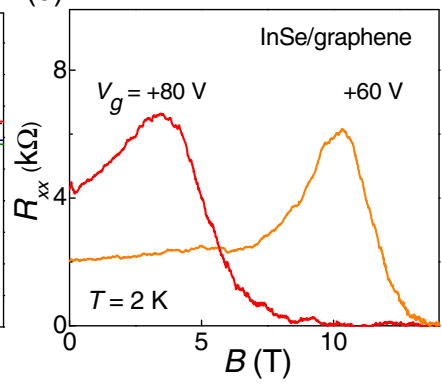

FIG. 1. Left: Crystal structure of InSe and graphene along the axis perpendicular to the layer plane. Right: Schematic and optical image of a Hall bar containing uncapped and InSe-capped graphene. (b) Hall resistance $R_{x y}$ for InSe/graphene and graphene versus magnetic field, $B$, for different gate voltages $V_{g}$ at temperature $T=2 \mathrm{~K}$. (c) Hall resistance $R_{x y}$ for InSe/graphene versus $B$ at different $T$ $\left(V_{g}=+80 \mathrm{~V}\right.$ and $\left.I=0.2 \mathrm{~mA}\right)$. (d) Magnetoresistance $R_{x x}$ for $\mathrm{InSe} /$ graphene versus $B$ for different $V_{g}$ at $T=2 \mathrm{~K}$.

The low-temperature $(T=2 \mathrm{~K})$ Hall resistance $R_{x y}$ and longitudinal magnetoresistance $R_{x x}$ of the capped and uncapped graphene layers were measured up to magnetic fields $B$ of $14 \mathrm{~T}$, applied perpendicular to the plane of the graphene layer and over a wide range of gate voltages $V_{g}$ applied between the graphene and the $\mathrm{Si}$ substrate. As shown in Fig. 1(b), the InSe-capped graphene layer reveals a broad QH resistance plateau with $R_{x y}=(12.9 \pm 0.1) \mathrm{k} \Omega$, which extends up to our maximum available $B$ of $14 \mathrm{~T}$. This value is close to that of the resistance quantum, $h / 2 e^{2}=12.906 \mathrm{k} \Omega$, corresponding to a Landau level filling factor $\nu=2$. In addition, at a large positive gate voltage [e.g., $V_{g}=+60 \mathrm{~V}$; see Fig. 1(b)], the $R_{x y}(B)$ curve exhibits a well-defined shoulder between 6 and $8 \mathrm{~T}$, with a resistance of $(4.3 \pm 0.1) \mathrm{k} \Omega$, close to the value $h / 6 e^{2}=$ $4.302 \mathrm{k} \Omega(\nu=6)$. In stark contrast, for uncapped graphene $R_{x y}$ increases linearly with $B$ up to $14 \mathrm{~T}$. The $\nu=2$ plateau persists up to a temperature of $T \sim 100 \mathrm{~K}$ [Fig. 1(c)], though with a smaller value of $R_{x y}$. In the vicinity of the $\nu=2$ plateau, $R_{x x}(B)$ approaches zero [Fig. 1(d)]. We have observed this behavior for several graphene layers capped with InSe [inset in Fig. 2(a)], although the QH resistance plateau is obtained for gate biases that vary slightly from sample to sample.

We use the Hall voltage data at low $B$ and low $T$ $(T=2 \mathrm{~K})$ to plot the $V_{g}$ dependence of the carrier density, $n$, for both the pristine graphene and the InSe-capped graphene layer [Fig. 2(a)]. Over the range of $V_{g}$ from -60 to $+60 \mathrm{~V}$, indicated as region I in Fig. 2(a), the measured hole density in both the uncapped and InSe-capped graphene decreases linearly with $V_{g}$ in agreement with the capacitance equation

$$
n=C V_{g} / q+n_{0} .
$$

Here $q=-e$ is the electron charge, $C=\varepsilon \varepsilon_{0} / t=$ $115 \mu \mathrm{F} / \mathrm{m}^{2}$ is the "classical" capacitance per unit area of the graphene $/ \mathrm{SiO}_{2} / \mathrm{Si}$ heterostructure, $t=300 \mathrm{~nm}$ is the $\mathrm{SiO}_{2}$ layer thickness, $\varepsilon=3.9$ is the relative dielectric constant of $\mathrm{SiO}_{2}, \varepsilon_{0}$ is the permittivity of free space, and $n_{0}=7.4 \times 10^{12}$ and $5.7 \times 10^{12} \mathrm{~cm}^{-2}$ are the hole densities at $V_{g}=0$ for the uncapped and capped graphene, respectively. Thus, the hole density for InSe-capped graphene, deduced from Hall measurements at $V_{g}=0$, is significantly smaller $\left(\Delta n=1.7 \times 10^{12} \mathrm{~cm}^{-2}\right)$ than for the uncapped control layer, indicating a "modulation doping" effect in which electrons are transferred from InSe to graphene. In contrast, over the range $+60 \mathrm{~V}<V_{g}<+100 \mathrm{~V}$ [see region II in Fig. 2(a)], the sheet density of the InSe-capped graphene deviates from the linear dependence on $V_{g}$ in Eq. (1), indicated by the dashed red line in Fig. 2(a). Furthermore, in region III $\left(V_{g}>+100 \mathrm{~V}\right)$, the conduction changes from $p$ to $n$ type and $n$ depends weakly on $V_{g}$.

The $V_{g}$ dependence of the low $T(T=2 \mathrm{~K})$ magnetoresistance $R_{x x}$ of the uncapped and InSe-capped graphene was measured at $B=0$; see Fig. 2(b). In both cases, $R_{x x}$ reaches a maximum at a large positive gate voltage, as expected for the $p$-type character of CVD graphene on $\mathrm{SiO}_{2}$ [15]. For InSe-capped graphene, the maximum of $R_{x x}\left(V_{q}\right)$ occurs at a lower gate voltage, shifted by $\Delta V_{g}=-15 \mathrm{~V}$ 


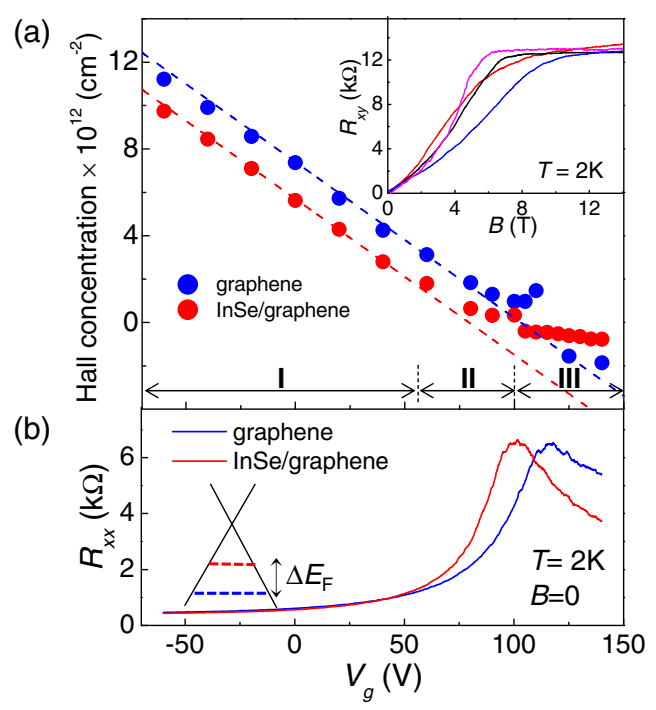

FIG. 2. (a) Hall carrier density in graphene and InSe/graphene versus gate voltage $V_{g}$ at $T=2 \mathrm{~K}$. Regions I, II, and III indicate different regimes of applied gate voltages. Blue and red lines correspond to the carrier density calculated from the capacitance equation (1) in the text. Inset: QH plateau for different InSe/ graphene samples at $T=2 \mathrm{~K}$. (b) Resistance $R_{x x}$ of graphene and InSe-capped graphene versus $V_{g}$ at zero magnetic field and $T=2 \mathrm{~K}$. Inset: Band diagram of graphene showing the upward shift of the Fermi level $E_{F}$ following its capping by InSe.

relative to the peak for uncapped graphene. The maximum of $R_{x x}\left(V_{g}\right)$ corresponds to the alignment of the Fermi energy $E_{F}$ with the Dirac point of graphene's band structure. For $V_{g}$ values beyond this maximum, the sign of the Hall voltage is reversed, corresponding to $n$-type conduction.

From the measured conductance $\sigma$ at $B=0$ and the Hall resistance $R_{x y}$, we obtain the Hall mobility $\mu_{H}$ using the relation $\mu_{H}=\sigma R_{x y}$, where $R_{x y}$ is extracted for low $B$ $(-2 \mathrm{~T}<B<2 \mathrm{~T})$ at different $V_{g}, \sigma=f / R_{x x}\left(V_{g}\right)$, and $f=1.5$ is the length-width ratio of the Hall bar. The $V_{g}$ dependence of $\mu_{H}$ is shown in Fig. 3: The data demonstrate that the InSe capping layer enhances significantly the carrier mobility in the graphene layer at all $V_{g}$, with an enhancement factor by up to $\sim 3$ at voltages that are close to the $V_{g}$ position of the peak in $R_{x x}\left(V_{g}\right)$. For InSe-capped graphene, the Hall mobility $\mu_{H}$ is also significantly larger than the field-effect mobility estimated using $\mu_{F}=\sigma / e n$, where $n\left(V_{g}\right)$ is given by the capacitance equation (1) (Supplemental Material Fig. S1 [16]).

The extended $\mathrm{QH}$ plateau with $\nu=2$ observed in the InSe/graphene $/ \mathrm{SiO}_{2}$ heterostructure [Fig. 1(b)] is similar in form to that reported for graphene grown epitaxially by the thermal decomposition of the surface layer of $\mathrm{SiC}$ [9-14]. Because graphene on $\mathrm{SiC}$ can maintain a $\mathrm{QH}$ state up to higher critical current densities and higher temperatures than GaAs-based heterostructures at comparable magnetic fields, it is regarded as an ideal candidate for

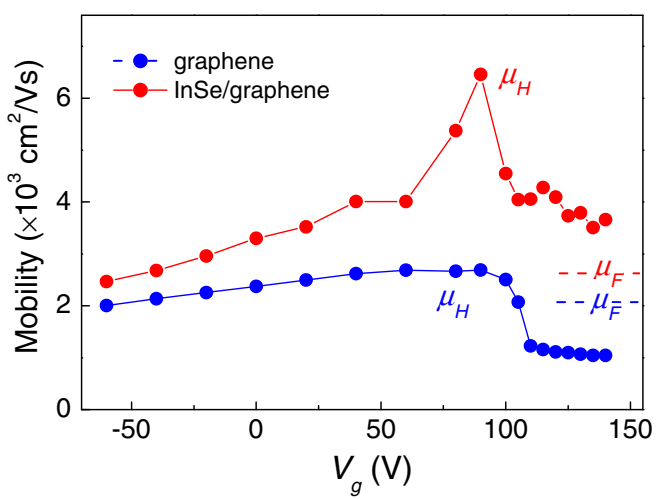

FIG. 3. Hall mobility $\mu_{H}$ (dots) and field-effect mobility $\mu_{F}$ (dashed lines) of uncapped and InSe-capped graphene at $T=2 \mathrm{~K}$. The Hall mobility $\mu_{H}$ is measured at different applied gate voltages $V_{g} \cdot \mu_{F}$ is estimated by modeling the measured $R_{x x}\left(V_{g}\right)$ curve. Continuous lines are guides to the eye.

the next generation of $\mathrm{QH}$ resistance standards [13-14]. The remarkable giant $\nu=2 \mathrm{QH}$ plateau in graphene on $\mathrm{SiC}$ has been explained in terms of the transfer of charge carriers from the underlying $\mathrm{SiC}$ substrate to the graphene layer, so that the Landau level at the Dirac point remains completely filled when its degeneracy, $2 e B / h$, increases with increasing $B[14,17]$.

Whereas it is difficult to achieve gate voltage control of graphene on $\mathrm{SiC}$, the design of our device provides a means of controlling and studying charge transfer across the InSe-graphene interface by applying a gate voltage to the $n$-Si substrate. In order to understand the electron transfer process in our device, we consider the band alignment at the InSe/graphene interface taking into account the electron affinities and work functions of InSe and graphene. For simplicity, we assume that the effect of defects introduced by the InSe layer is negligible. This assumption is reasonable, as the cleaved surface of InSe contains a low density of surface states and the layers are functional optically and electrically down to a few atomic layers [8]. Nevertheless, we do not exclude that a small density of defects may form during the device fabrication and that this may affect the charge transfer.

The electron affinity of graphene $\left(\chi_{\mathrm{Gr}}=4.5 \mathrm{eV}\right)$ is only slightly smaller than that of bulk InSe $\left(\chi_{\mathrm{InSe}}=4.6 \mathrm{eV}\right)$ $[7,18]$. These values of $\chi$ and the measured concentration of carriers in bulk InSe (electron density $\sim 10^{15} \mathrm{~cm}^{-3}$ ) and the uncapped graphene (hole density $\sim 7 \times 10^{12} \mathrm{~cm}^{-2}$ ) indicate that at $V_{g}=0$ the work function of our CVD graphene on $\mathrm{SiO}_{2}\left(\phi_{\mathrm{Gr}} \approx 4.8 \mathrm{eV}\right)$ is larger than that of the $n$-type InSe $\left(\phi_{\text {InSe }} \approx 4.7 \mathrm{eV}\right)$. At $V_{g}=0$, equilibrium is achieved by a transfer of electrons from the $n$-InSe to graphene. This electron transfer process is evident from the data shown in Fig. 2. It induces a depletion layer and an upward bend of the InSe CB edge near its interface with graphene, shown schematically in Fig. 4(a). Also, photoluminescence studies of InSe flakes with and without an underlying graphene 


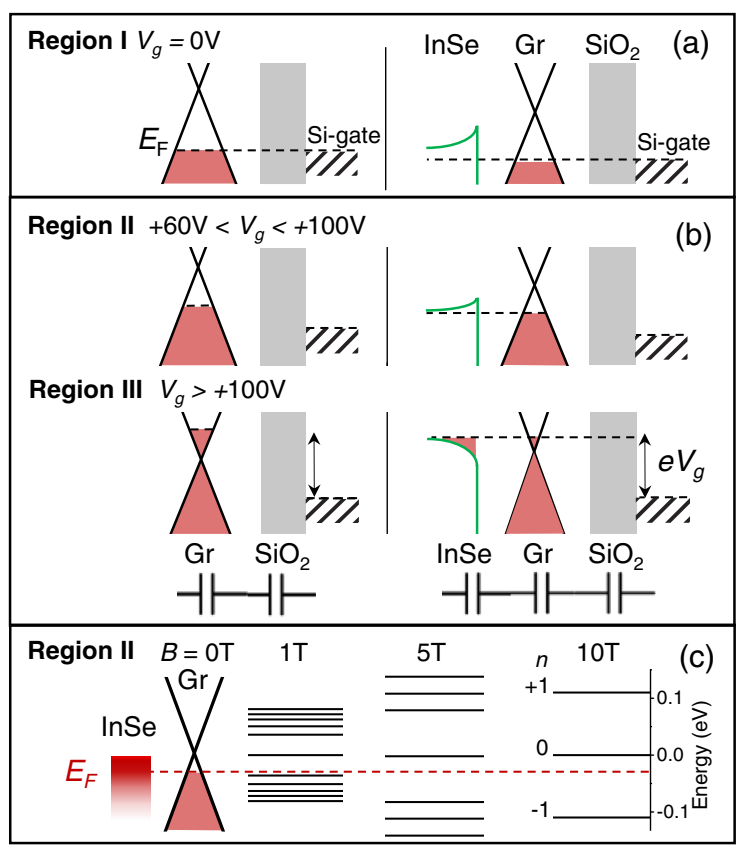

FIG. 4. Energy band diagram of the graphene $/ \mathrm{SiO}_{2} / \mathrm{Si}$ and $\mathrm{InSe} /$ graphene $/ \mathrm{SiO}_{2} / \mathrm{Si}$ heterostructure at different gate biases $V_{g}$ corresponding to (a) region I $\left(V_{g}=0 \mathrm{~V}\right)$ and (b) regions II $\left(60 \mathrm{~V}<V_{g}<100 \mathrm{~V}\right)$ and III $\left(V_{g}>100 \mathrm{~V}\right)$. The red regions indicate states filled with electrons. The green curves indicate the band bending in InSe. The bottom inset in part (b) illustrates the capacitance contributions to the total capacitance of the heterostructures. (c) Landau levels of graphene at $B=0,1,5$, and $10 \mathrm{~T}$ (region II). For InSe-capped graphene, the Fermi level is pinned between the $n=0$ and $n=-1$ Landau levels over a wide range of $B$.

layer indicate that the optical emission for the InSe flakes on graphene is weaker, suggestive of a charge transfer at the InSe/graphene interface (Supplemental Material Fig. S2 [16]).

We use the equivalent circuit shown in Fig. 4(b) (lower right inset) to model the electrostatics of the device, noting that the chemical potentials of the graphene and InSe layers remain aligned in the heterostructure at all $V_{g}$ and $B$ because no voltage is applied between them. The total capacitance $C_{t}$ of the InSe/graphene $/ \mathrm{SiO}_{2} / \mathrm{Si}$ heterostructure is a series combination of the classical capacitance $C_{c}$ of the $\mathrm{SiO}_{2}$ and the InSe depletion layer and the quantum capacitance $C_{q}$ of graphene: $C_{t}^{-1}=C_{c}^{-1}+C_{q}^{-1}$. The quantum capacitance accounts for the nonclassical dependence of graphene's carrier density, $n$, on the applied gate voltage when the Fermi energy $E_{F}$ is close to the low density of states at the Dirac point: $C_{q}=e^{2}\left(d n / d E_{F}\right)=2 e^{2} E_{F} / \pi \hbar^{2} v_{F}^{2}$. When the Fermi level is far from the Dirac point, the effect of the quantum capacitance becomes negligible, and the carrier density in graphene varies with $V_{g}$ according to the capacitance equation (1), i.e., $q d n=C_{c} d V_{g}$, as observed experimentally in region I of Fig. 2(a). The measured values of the field-effect mobility $\mu_{F}=\sigma / e n$ and the Hall mobility $\mu_{H}=\sigma R_{x y}$ are also in qualitative agreement (Fig. 3, $V_{g}<+60 \mathrm{~V}$ ). In contrast, when the positive gate voltage is sufficiently large so that $E_{F}$ approaches the Dirac point, the total capacitance is reduced due to the small quantum capacitance, leading to a weaker variation of $n$ with $V_{g}$.

The slope of the measured $n\left(V_{g}\right)$ dependence for InSecapped graphene changes twice between $V_{g}=+60$ and $+100 \mathrm{~V}$ [i.e., the beginning and end of region II, Fig. 2 (b)]. This behavior indicates a regime in which the upward bend of the InSe CB edge is reduced due to the positive space charge of ionized donors and electrons start to fill donor states below the $\mathrm{CB}$ edge of InSe [region II, Fig. 4(b)]. The Fermi energy aligns with the Dirac point at $V_{g} \approx+100 \mathrm{~V}$ [Fig. 2(b)]. The change in the slope of $n\left(V_{g}\right)$ tends to saturate for $V_{g}>+100 \mathrm{~V}$ [region III, Fig. 2(a)]. We attribute this to the movement of the Fermi level into the high density of states in the CB continuum of InSe, leading eventually to the formation of an electron accumulation layer at high positive $V_{g}$ [region III, Fig. 4(b)]. Since the density of states of graphene at energies close to the Dirac point is much lower than in the $\mathrm{CB}$ of InSe, the chemical potential tends to remain pinned near the InSe CB edge. The gate voltages $V_{g}=+60$ and $+100 \mathrm{~V}$, at which the slope of the measured $n\left(V_{g}\right)$ dependence changes can be used to estimate the binding energy of the donor states in InSe. These are around $50 \mathrm{meV}$ below the $\mathrm{CB}$ edge (Supplemental Material Fig. S3 [16]), in qualitative agreement with results of optical studies [19].

The favorable band alignment between InSe and graphene is an important factor that enables effective modulation doping of graphene over a wide range of gate biases. The appearance of the extended $\nu=2 \mathrm{QH}$ plateau relies on this charge transfer and on the "pinning" of the chemical potential in the energy gap between the $n=0$ and $n=1$ Landau levels when their degeneracy increases with increasing $B$ [Fig. 4(c)]. The extended $\nu=2$ plateau persists up to $T \sim 100 \mathrm{~K}$, though with a smaller value of $R_{x y}$, which decreases with increasing $T$ [Fig. 1(c)]. We attribute this decrease to thermally activated parallel conduction along the InSe capping layer. Using a model based on two resistances "in parallel" [20], we express the measured Hall conductance $R_{x y}^{-1}$ as $R_{x y}^{-1}=R_{\mathrm{InSe}}^{-1}+2 e^{2} / h$, where $R_{\mathrm{InSe}}=e n_{\mathrm{InSe}} / B$ is the Hall resistance of the InSe layer and $h / 2 e^{2}$ is the $\mathrm{QH}$ resistance of graphene. Our data indicate that parallel conduction can be neglected at $T=2 \mathrm{~K}$; also, we estimate that the carrier density in the InSe layer, $n_{\text {InSe }}$, increases from $\sim 10^{9} \mathrm{~cm}^{-2}$ at $T=$ $2 \mathrm{~K}$ to $\sim 10^{11} \mathrm{~cm}^{-2}$ for $T>100 \mathrm{~K}$; correspondingly, $R_{\text {InSe }}$ decreases from $\sim 90 \mathrm{M} \Omega$ to $90 \mathrm{k} \Omega$ (Supplemental Material Fig. S4 [16]).

The significant increase of carrier mobility in region II (Fig. 3) can be explained by partial screening of the negatively 
charged (defect) acceptors at the $\mathrm{SiO}_{2} /$ graphene interface by positively charged donors in InSe and by the dielectric screening due to the static dielectric constant of InSe $\left(\varepsilon_{\text {InSe }} \approx 10\right.$ [21]). In CVD graphene on $\mathrm{SiO}_{2}$, the main mechanism limiting the carrier mobility is electron scattering by negatively charged acceptors. It has been described phenomenologically by the relation $\mu=\alpha\left(N_{0} / N\right)$, where $N$ is the concentration of the charged acceptors and $N_{0}$ and $\alpha$ are empirical parameters [22]. Our measured enhancement of mobility in the InSe-capped graphene layer indicates an electrostatic compensation of these charged acceptor impurities. This effect is analogous to that observed recently in $p$-type CVD graphene decorated with $n$-type quantum dots (QDs), where the largest increase of mobility is observed when the concentrations of donors in the QDs matches the concentration of impurities in graphene [15]. However, an extended QH plateau was never reported for graphene capped with QDs. This difference can be attributed to the absence of Fermi level pinning due to the low density of states in the QDs compared to that in the $\mathrm{CB}$ of InSe.

In conclusion, we have reported on a giant $\mathrm{QH}$ plateau in monolayer graphene capped with InSe. The favorable alignment of the band structure of these two materials enables a transfer of electrons across their vdW interface. This modulation doping of graphene is controlled by applying a gate voltage to the doped $\mathrm{Si}$ substrate and leads to an extended $\nu=2 \mathrm{QH}$ plateau. Prospects for further research include the investigation of the charge transfer for different InSe layer thicknesses and the optimization of the $\mathrm{QH}$ plateau by further advances in the design of multilayered $\mathrm{vdW}$ heterostructures. InSe/graphene therefore represents a novel heterostructure for exploring the giant $\mathrm{QH}$ plateau phenomenon, with potential for metrological applications and for future technologies that exploit modulation doping and controlled charge transfer between the component layers of vdW heterostructures.

The data on which this manuscript is based are available as an online resource with digital object identifier [23].

This work was supported by the Engineering and Physical Sciences Research Council [Grant No. EP/M012700/1] (EPSRC); the EU FP7 Graphene Flagship Project No. 604391; The University of Nottingham; The National Academy of Sciences of Ukraine; and RFBR [15-02-01221 and 17-02-01129]. We acknowledge A.J. Marsden and N. R. Wilson (University of Warwick) for providing us with CVD graphene.
* Corresponding author.

oleg.makarovsky@nottingham.ac.uk

Corresponding author. amalia.patane@nottingham.ac.uk

[1] A. C. Ferrari et al., Nanoscale 7, 4598 (2015).

[2] G. W. Mudd et al., Adv. Mater. 25, 5714 (2013).

[3] M. Brotons-Gisbert et al., Nano Lett. 16, 3221 (2016).

[4] G. W. Mudd et al., Sci. Rep. 6, 39619 (2016).

[5] W. Feng, W. Zheng, W. Cao, and P. A. Hu, Adv. Mater. 26, 6587 (2014).

[6] R. T. Srinivasa, Y.-Y. Lu, U. Rajesh Kumar, R. Sankar, C.-D. Liao, B. Karukanara Moorthy, C.-H. Cheng, F. C. Chou, and Y.-T. Chen, Nano Lett. 14, 2800 (2014).

[7] G. W. Mudd et al., Adv. Mater. 27, 3760 (2015).

[8] D. A. Bandurin et al., Nat. Nanotechnol. 12, 223 (2017).

[9] A. Tzalenchuk, S. Lara-Avila, A. Kalaboukhov, S. Paolillo, M. Syväjärvi, R. Yakimova, O. Kazakova, T. J. B. M. Janssen, V. Fal'ko, and S. Kubatkin, Nat. Nanotechnol. 5, 186 (2010).

[10] S. Kopylov, A. Tzalenchuk, S. Kubatkin, and V. I. Fal'ko, Appl. Phys. Lett. 97, 112109 (2010).

[11] T. J. B. M. Janssen, A. Tzalenchuk, S. Lara-Avila, S. Kubatkin, and V. I. Fal'ko, Rep. Prog. Phys. 76, 104501 (2013).

[12] R. Ribeiro-Palau et al., Nat. Nanotechnol. 10, 965 (2015).

[13] T. J. B. M. Janssen, S. Rozhko, I. Antonov, A. Tzalenchuk, J. M Williams, Z. Melhem, H. He, S. Lara-Avila, S. Kubatkin, and R. Yakimova, 2D Mater. 2, 035015 (2015).

[14] J. A. Alexander-Webber et al., Sci. Rep. 6, 30296 (2016).

[15] L. Turyanska, O. Makarovsky, L. Eaves, A. Patanè, and N. Mori, 2D Mater. 4, 025026 (2017).

[16] See Supplemental Material at http://link.aps.org/ supplemental/10.1103/PhysRevLett.119.157701 for the field effect mobility, the effect of graphene on the photoluminescence of InSe, the donor energy in $n$-type InSe, and the parallel conduction in InSe.

[17] T. J. B. M. Janssen, A. Tzalenchuk, R. Yakimova, S. Kubatkin, S. Lara-Avila, S. Kopylov, and V. I. Falko, Phys. Rev. B 83, 233402 (2011).

[18] Electron Spec. Appl. to Low-Dimensional Materials, edited by H.P. Hughes and H. I. Starnberg (Kluwer Academic Dordrecht, 2000), p. 317.

[19] G. W. Mudd, A. Patanè, Z. R. Kudrynskyi, M. W. Fay, O. Makarovsky, L. Eaves, Z. D. Kovalyuk, V. Zólyomi, and V. Falko, Appl. Phys. Lett. 105, 221909 (2014).

[20] M. Reed, W. P. Kirk, and P. S. Kobiela, IEEE J. Quantum Electron. 22, 1753 (1986).

[21] O. Madelung, U. Rössler, and M. Schulz, NonTetrahedrally Bonded Elements and Binary Compounds I (Springer, Berlin 1998).

[22] S. Adam, E. H. Hwang, V. M. Galitski, and V. DasSarma Proc. Natl. Acad. Sci. U.S.A. 104, 18392 (2007).

[23] DOI: 10.17639/nott.323. 